\title{
Heart failure patients with prior right ventricular pacing upgraded to resynchronization therapy had a worse prognosis compared to de novo cardiac resynchronization therapy at 1-year follow-up. Results from a Chagas disease cohort
}

\author{
RODRIGO VIEIRA DE MELO ${ }^{1}$, William Carvalho ${ }^{2}$, Tainá Viana ${ }^{1}$, Diogo Azevedo², \\ Cristiano Bezerra ${ }^{2}$, Clara Figueiredo ${ }^{1}$, and Luiz Carlos Passos ${ }^{1}$ \\ ${ }^{1}$ Universidade Federal da Bahia \\ ${ }^{2}$ Hospital Ana Nery
}

June 1, 2020

\begin{abstract}
Introduction: Cardiac resynchronization therapy (CRT) improves outcomes in heart failure (HF) patients with left bundle branch block (LBBB). However, the benefits of CRT in patients with previous pacing are uncertain, specially in a population witch Chagas disease is a prevalent cause of HF. Methods and Results: Prospective cohort study that included HF patients indicated for CRT with left ventricular ejection fraction (LVEF) of less than 35\%. Clinical and demographic data were collected to investigate mortality predictors after 1 year. The overall survival was calculated by the Kaplan-Meier method and multivariate analysis using Cox's regression model was performed. Between May 2017 and September 2019, 93 patients were evaluated with a mean follow-up of $1,0(0.6)$ year. Of these, $22(23,7 \%)$ were upgraded from right ventricular pacing. Chagas Disease was the most prevalent cause of HF $29(31,2 \%)$. In overall patients, LVEF at 6 months increased after CRT: $24,0 \%(7,8)$ to $30,3 \%(11,5)$, $\mathrm{p}=0.007$, and there was no significant difference between upgraded patients and de Novo CRT, $\mathrm{p}=0.26$. Overall mortality at 1-year was $28(30,1 \%)$. In the univariate analysis, Chagas disease and upgraded therapy were associated with mortality at follow-up, HR: 3.9 , CI: $1.8-8,4, \mathrm{p}=0.001$ and HR: 4.7, CI: 2.2-9.9, p $<0.001$, respectively. In the multivariate model, only upgraded therapy remained independently associated with the outcome, adjusted HR: 2.9, CI: 1.2-7,2, p = 0.02. Conclusion: In this specific HF population, with a high prevalence of Chagas disease cardiomyopathy, upgraded therapy was independently associated with worsened 1-year survival after CRT.
\end{abstract}

\section{Introduction}

Randomized controlled trials supports the clinical efficacy and safety of cardiac resynchronization therapy (CRT) in patients with moderate or severe heart failure and ventricular dyssynchrony. ${ }^{1,2}$ Guidelines from international cardiology societies provide strong recommendations for CRT specially in symptomatic patients with left bundle branch block (LBBB) and a QRS duration $>150 \mathrm{~ms} .{ }^{3}$ However, important questions still remain about the clinical application of this therapy in specific populations.

Upgrade for a CRT from a conventional pacemaker have become increasingly common in HF patients, since right ventricular pacing may aggravate left ventricular function. ${ }^{4}$ Despite this, concerns persist as this conduct is supported by small and observational studies. In this sense, recent evidences suggest that clinical response and survival are impaired in patients undergoing CRT upgrade compared to de novo implantations. ${ }^{5,6}$

Chagas disease (CD) cardiomyopathy remains a prevalent cause of HF in Latin America, despite this, it is underrepresented in most CRT-trials. It is well established that CD patients had a worse prognosis compared 
to other HF etiologies, and recent studies suggests that these patients also have a higher short-term mortality rate after CRT. ${ }^{7,8}$ As the need for a pacemaker implantation is common in the course of CD cardiomyopathy, and since LBBB is relatively rare in this patients, an expected higher incidence of upgrade-CRT implantation in this population might play a role in worsening the outcomes.

Thus, the aim of this study is to compare the short-term mortality between upgrade and de Novo-CRT implantation in a heart failure population where Chagas disease is endemic.

\section{Methods}

\section{Population}

Prospective cohort study between May 2017 and September 2019. We included consecutive outpatients over 18 years, followed at the heart failure unit of the Ana Nery Hospital of the Federal University of Bahia in Brazil. The indication for CRT was based on the following criteria: patients age over 18 years, under appropriate medical treatment, presenting NYHA II to IV with left ventricular ejection fraction (LVEF) less than $35 \%$ and a QRS duration > 150ms or $120-150 \mathrm{~ms}$ with proven dyssynchrony. Patients with previously implanted pacemakers or implantable cardioverter-defibrillators (ICD) who developed this criteria, with or without need for continuous ventricular pacing, were also considered for CRT (upgrade group).

Demographic, laboratory and echocardiographic data were collected at the time of the hospitalization for the procedure. Left ventricular ejection fraction was measured on transthoracic echocardiograms using the Simpson's method at the time of the CRT implantation, and after 6 months. Chronic renal disease was defined as renal clearance, estimated using Cockroft and Gault's formula, $<60 \mathrm{~mL} / \mathrm{min} / 1.73 \mathrm{~m} 2$. Atrial fibrillation was defined at the time of the procedure by baseline electrocardiogram. Chagas disease was confirmed by specific serological tests.

Patients were excluded if they had a chronic systemic inflammatory disease, malignant neoplasia under treatment, patients with no clear etiology of heart failure, or who refused the procedure or declined to give informed consent.

\section{Follow-up and outcomes}

Patients were followed through regular outpatient visits at the institution after hospital discharge. Those patients who did not return within 1 year after CRT implantation were contacted by telephone. Survival was assessed as the time from CRT implantation to all-cause mortality.

\section{Ethics Committee}

The local ethics committee approved the study, and all procedures were performed according to the Helsinki statement.

\section{Statistical analysis}

The Kolmogorov-Smirnov test was used to verify the normal distribution of continuous variables. Variables with normal distribution were described by means and standard deviations and compared by Student's ttest. Categorical data were presented as the number of patients and percentage of the total sample and were compared by the Fisher's exact test. The overall survival was calculated by the Kaplan-Meier method and the log-rank test was used to compare the survival curves. A value of $\mathrm{P}<0.05$ was considered as statistically significant. To identify the variables that are independently predictive of overall mortality, a subsequent stepwise multivariate analysis using Cox's regression model was performed, including variables that had a predictive value of $\mathrm{P}$-value $<0.10$ in the univariate analysis.

\section{Results}

One hundred patients were evaluated for CRT implantation, seven of them were excluded due to a LVEF higher than $35 \%$ prior the procedure. Of the remaining 93 patients, Chagas Disease was the most prevalent cause of HF $29(31.2 \%)$, followed by idiopathic dilated cardiomyopathy with 28 (30.1\%) patients. There was 
no lost of follow-up for the main outcome, with a mean duration of 1,0 $( \pm 0.6)$ year. Patients upgraded from a right ventricular pacing (upgrade group) were $22(23.7 \%)$, of those, $4(18.2 \%)$ had previously an implantable cardioverter-defibrillator. Baseline demographic characteristics of groups upgrade and de novo are provided in Table 1.

Chagas disease cardiomyopathy was most prevalent in the upgraded patients: 16 (72.7\%) vs 13 (18.3\%), $\mathrm{p}<0.001$. The following variables were well balanced between upgrade and de novo groups: Atrial fibrillation $5(22.7 \%)$ vs $15(21.1 \%), \mathrm{p}=1.000$; chronic kidney disease $8(36.4 \%)$ vs $17(23.9 \%), \mathrm{p}=0.278$; LVEF: $22.3 \%$ $( \pm 7.1)$ vs $24.4 \%( \pm 7.6), \mathrm{p}=0.249$; NYHA class III-IV: 19 (86.4\%) vs $57(80.3 \%), \mathrm{p}=0.754$, respectively. Cardiac resynchronization therapy with defibrillator implantation (CRT-D) rates were: 14 (63.6\%) in upgraded group and $29(40.8 \%)$ in de novo group, $\mathrm{p}=0.086$.

Medical treatment for heart failure with evidence-based medical therapies were optimized in both groups: beta-blocker: 21 (95.5\%) vs $65(91.5 \%), \mathrm{p}=1.000$; angiotensin-converting enzyme inhibitors or angiotensin receptor blockers or angiotensin receptor neprilysin inhibitor: 20 (90.9\%) vs 61 (85.9\%), p=0.725; and aldosterone receptor antagonists: $18(81.8 \%)$ vs $63(88.7 \%), \mathrm{p}=0.847$; for upgrade and de novo group respectively.

Pairwise echocardiographic measurements (baseline and 6 months of follow-up) were available in 78 (83.9\%) patients. Both groups improved the LVEF on the 6-month echocardiogram: $22.3 \%( \pm 7.1)$ to $27.1 \%( \pm 9.5)$, $\mathrm{p}<0.001$ and $24.4 \%( \pm 7.6)$ to $31.1 \%( \pm 11.9), \mathrm{p}<0.001$, for upgrade and de novo , respectively, but there was no difference of $\triangle \mathrm{LVEF}$ improvement between groups, $\mathrm{p}=0.246$. No patient underwent heart transplantation during the study period.

In the follow-up, overall mortality occurred in 28 (30.1\%) patients, with more frequent death in upgraded patients when compared to de novo CRT implantation, 14 (63.6\%) vs $14(19.7 \%)$, p < 0.001 (log rank), figure 1. There were four in-hospital deaths, all of them directly associated with the procedure and all belonging to the upgraded group. In the univariate analysis, Chagas disease and upgraded therapy were associated with overall mortality at follow-up, HR: 3.9, CI: 1.8-8,4, p = 0.001 and RR: 4.7, CI: 2.2-9.9, p < 0.001 , respectively. In the multivariate model including both variables, and combined therapy with CRT-D, only upgraded therapy remained independently associated with the outcome, adjusted HR: 2.9, CI: 1.2-7,1, $\mathrm{p}=0.019$ ), Table 2 .

\section{Discussion}

We present a prospective cohort of patients with heart failure and an indication for CRT where Chagas disease was the most frequent cause of cardiomyopathy. There was no loss of follow-up and use of evidence-based medical therapies was higher than most previous CRT-trials. ${ }^{9,10}$ Furthermore, the indication for CRT was consistent with guideline-based recommendations, with almost all patients presenting with LBBB (induced or spontaneous) and QRS>150ms.

Patients undergoing the upgraded CRT implantation had similar clinical, echocardiographic and demographic characteristics when compared to the population of new CRT, only differing with a higher prevalence of Chagas' disease. Unlike previous studies comparing de novo CRT vs upgraded, we found no difference regarding the prevalence of atrial fibrillation or QRS duration between both groups. ${ }^{5,11}$

Overall population was a high-risk one, with LVEF in average below $25 \%$ and most patients on NYHA class III or IV. In general, CRT was effective in improving systolic performance with a significant increase in LVEF. This left ventricular reverse remodeling occurred consistently in both groups, upgraded and de novo . This result is in line with a recently published meta-analysis that demonstrated similar rates of improvement in LVEF in patients undergoing upgraded and de novo -CRT. ${ }^{12}$

However, we observed a high overall mortality in 1-year follow-up of $30.1 \%$, mainly in the upgraded-CRT group. In univariate analysis, Chagas disease and upgraded-CRT were directly associated with overall mortality in 1-year, and the main find of our study was that in the multivariate model, upgraded therapy was the solely variable associated with the outcome. This finding, and the excessive mortality-rate in the upgraded group, is consistent with the study of Vamos M et al, that followed 552 CRT implantations, 
including 177 upgrade procedures, and found a 1.65-fold increased mortality. Similarly, the cohort of Beca $\mathrm{B}$ et al, found a long-term mortality rate 2.86 -fold increased. On the other hand, this data differs from previously mentioned meta-analysis, and the European CRT survey, that demonstrated that CRT upgrade is associated with similar risk for all-cause mortality compared to de novoresynchronization therapy. ${ }^{5,11-13}$

Some factors may justify these findings, firstly, it has been suggested that CRT upgrade procedures are associated with increased peri-procedural complications. In fact, all in-hospital deaths occurred in the upgraded-CRT group, and directly related to the procedure. Our sample size was not sufficient to test this hypothesis. Data comparing the rates of complications following CRT upgrade versus de novoCRT are limited and inconsistent. In a large European CRT Survey of 11088 patients, and 2396 (23.2\%) upgrade procedures, overall peri-procedural complication rates were similar between upgraded-CRT and de novo CRT. In contrast, Cheung JW et al, using the United States National database, identified a significantly higher rate of complications in CRT upgrade patients compared to de novo CRT patients with a two-fold increased risk of in-hospital mortality. ${ }^{6,14}$

Other hypothesis is that patients in the upgrade group had more advanced heart disease and more comorbidities, and the indication for biventricular pacing may have been initiated too late. In our series, upgraded patients had similar baseline characteristics of advanced heart failure compared to de novo patients. However, despite the lack of statistical significance, upgraded patients had a trend to be older, with slightly lower left ventricular ejection fraction and higher prevalence of NYHA III and IV.

Finally, particularly in this heart failure population, Chagas disease might play a role in impairing patients survival. The pathophysiological and epidemiological characteristics of Chagas disease itself corroborate worse outcomes. Heart failure patients due to Chagas cardiomyopathy are known to have a worse prognosis compared to other etiologies, with a higher incidence of death from heart failure progression and arrhythmic death. ${ }^{15-17}$. Particularly in patients undergoing CRT, it has been consistently demonstrated that Chagas cardiomyopathy has a worse prognosis when compared to other heart failure etiologies. Martinelli et al showed that Chagas disease had a two-fold higher risk of death in one-year compared to the others dilated cardiomyopathy. Simirlaly, Passos, et al also demonstrated a worse prognosis in combined events in patients with Chagas cardiomyopathy after CRT. ${ }^{7,8}$

It is important to emphasize that, since intrinsic LBBB in Chagas heart disease is uncommon, and it is considered an arrhythmogenic cardiomyopathy characterized by a wide variety of abnormalities of the conduction, it is expected a higher incidence of upgraded-CRT implantation in this patients. In fact, in the cohort presented by Martinelli et al, there was a $73.9 \%$ incidence of induced-LBBB in Chagas' disease patients undergoing CRT. In our study, $72.7 \%$ of patients undergoing upgraded-CRT implantation had Chagas cardiomyopathy.

This is the first study in which we are known to specifically address the impact of the upgraded-CRT on mortality in a population where Chagas disease is a prevalent cause of cardiomyopathy. In fact, in line with previous publications, Chagas' disease was directly associated with an increase in short-term mortality after CRT implantation, however after multivariate analysis adjusted for potential confounders, it is suggested that this worse prognosis is due to the higher incidence of upgraded-CRT in this patients. Studies with larger cohorts of Chagas cardiomyopathy patients are necessary to confirm this hypothesis. Until then, considering the current scientific evidence, patients with heart failure secondary to Chagas disease and previous ventricular pacing, must have the indication for the upgrade procedure evaluated with great caution.

This study has some limitations. We emphasize the unicentric design of the study, which may impact in its external validity. Additionally, it is a non-randomized study that generates hypothesis and is exposed to confounding bias. Finally, the limited sample size makes the study vulnerable to type 1 error.

\section{Conclusion}

In this heart failure cohort of patients with high prevalence of Chagas disease cardiomyopathy, survival was less-favorable in patients undergoing CRT upgrade compared to de novo implantations. Until further 
evidence is available, this result should be taken into account when evaluating upgrade therapy in Chagas disease patients with previous ventricular pacing.

\section{References:}

1. Cleland JG, Abraham WT, Linde C, Gold MR, Young JB, Claude Daubert J, et al. An individual patient meta-analysis of five randomized trials assessing the effects of cardiac resynchronization therapy on morbidity and mortality in patients with symptomatic heart failure. Eur Heart J. 2013;34:35473556. doi: 10.1093/eurheartj/eht290

2. Zareba W, Klein H, Cygankiewicz I, Hall WJ, McNitt S, Brown M, et al; MADIT-CRT Investigators. Effectiveness of cardiac resynchronization therapy by QRS Morphology in the Multicenter Automatic Defibrillator Implantation Trial-Cardiac Resynchronization Therapy (MADIT-CRT). Circulation. 2011;123:1061-1072. doi: 10.1161/ CIRCULATIONAHA.110.960898.

3. Normand C, Linde C, Singh J, Dickstein K. Indications for cardiac resynchronization therapy: a comparison of the major international guidelines. JACC Heart Fail 2018;6:308-316.

4. Curtis AB, Worley SJ, Adamson PB, Chung ES, Niazi I, Sherfesee L, Shinn T, Sutton MS; Biventricular versus Right Ventricular Pacing in Heart Failure Patients with Atrioventricular Block (BLOCK HF) Trial Investigators. Biventricular pacing for atrioventricular block and systolic dysfunction. N Engl J Med 2013;368:1585-1593.

5. Vamos M, Erath JW, Bari Z, Vagany D, Linzbach SP, Burmistrava T, Israel CW, Duray GZ, Hohnloser SH (2017). Effects of upgrade versus de novo cardiac resynchronization therapy on clinical response and long-term survival: results from a multicenter study. Circ Arrhythmia Electrophysiol 10(2):e004471. https://doi.org/10.1161/circep.116.004471

6. Cheung JW, Ip JE, Markowitz SM, Liu CF, Thomas G, Feldman DN, et al. Trends and outcomes of cardiac resynchronization therapy upgrade procedures: a comparative analysis using a United States National Database 2003-2013. Heart Rhythm (2017), doi: 10.1016/j.hrthm.2017.02.017.

7. Martinelli Filho M, Peixoto GL, Siqueira SF, Nishioka ASD, Pedrosa ALA, Teixeira RA. A cohort study of cardiac resynchronization therapy in patients with chronic Chagas cardiomyopathy. Europace 2018;20:1813-8.

8. Passos LCS, Vieira de Melo RM, Lira YM, Oliveira NFC, Trindade TC, Carvalho W, et al . Chagas disease is associated with a poor outcome at 1-year follow-up after cardiac resynchronization therapy. Rev. Assoc. Med. Bras. [Internet]. 2019 Nov [cited 2020 May 12] ; 65( 11 ): 1391-1396. https://doi.org/10.1590/1806-9282.65.11.1391.

9. St. John Sutton MG, Plappert T, Abraham WT, Smith AL, DeLurgio DB, Leon AR, et al. Effect of cardiac resynchronization therapy on left ventricular size and function in chronic heart failure. Circulation. 2003;107(15):1985-90.

10. Moss AJ, Hall WJ, Cannom DS, Klein H, Brown MW, Daubert JP, et al. Cardiac-Resynchronization Therapy for the Prevention of Heart-Failure Events. N Engl J Med. 2009;361(14):1329-38.

11. Bogale N, Witte K, Priori S, Cleland J, Auricchio A, Gadler F, et al. The European Cardiac Resynchronization Therapy Survey: comparison of outcomes between de novo cardiac resynchronization therapy implantations and upgrades. Eur J Heart Fail 2011;13:974-83.

12. Kosztin A, Vamos M, Aradi D, Schwertner WR, Kovacs A, Nagy KV, et al. De novo implantation vs. upgrade cardiac resynchronization therapy: a systematic review and meta-analysis. Heart Fail Rev. 2018;23(1):15-26. doi:10.1007/s10741-017-9652-1.

13. Beca B, Sapp JL, Gardner MJ, Gray C, AbdelWahab A, MacIntyre C, et al. Mortality and Heart Failure After Upgrade to Cardiac Resynchronization Therapy. CJC Open. 2019;1(2):93-99. Published 2019 Mar 6. doi:10.1016/j.cjco.2019.02.002

14. Linde CM, Normand C, Bogale N, Auricchio A, Sterlinski M, Marinskis G, et al. Upgrades from a previous device compared to de novo cardiac resynchronization therapy in the European Society of Cardiology CRT Survey II. Eur J Heart Fail. 2018;20(10):1457-1468. doi:10.1002/ejhf.1235

15. Mady C, Cardoso RH, Barreto ACP, Da Luz PL, Bellotti G, Pileggi F. Survival and predictors of survival in patients with congestive heart failure due to Chagas' cardiomyopathy. Circulation. 1994; 
90: 3098-102.

16. Silva CP, Del Carlo CH, Oliveira MT Junior, Scipioni A, Strunz-Cassaro C, Ramirez JA, et al. Why do patients with chagasic cardiomyopathy have worse outcomes than those with non-chagasic cardiomyopathy? Arq Bras Cardiol. 2008;91(6):358-62.

17. Oliveira-Filho J, Viana LC, Vieira-de-Melo RM, Faiçal F, Torreão JA, Villar FA, et al.. Chagas disease is an independent risk factor for stroke: baseline characteristics of a Chagas Disease cohort. Stroke. 2005; 36:2015-2017.

Table 1 - Demographic data and baseline clinical characteristics

\begin{tabular}{|c|c|c|c|}
\hline & Upgrade group $n=22$ & de Novo group $\mathbf{n}=\mathbf{7 1}$ & $\mathrm{p}$ \\
\hline \multicolumn{4}{|l|}{ Heart failure etiology } \\
\hline Chagas, n (\%) & $16(72.7 \%)$ & $13(18.3 \%)$ & $<0.001$ \\
\hline Idiopathic, n (\%) & $1(4.5 \%)$ & $27(38.0 \%)$ & 0.003 \\
\hline Ischemic, n (\%) & $1(4.5 \%)$ & $14(19.7 \%)$ & 0.109 \\
\hline Age, years $( \pm \mathrm{SD})$ & $62.4( \pm 13.8)$ & $56.6( \pm 11.4)$ & 0.080 \\
\hline Male gender, n (\%) & $15(68.2 \%)$ & $36(50.7 \%)$ & 0.220 \\
\hline $\mathrm{LVEF}$, mean $( \pm \mathrm{SD})$ & $22.3( \pm 7.1)$ & $24.4( \pm 7.6)$ & 0.249 \\
\hline $\begin{array}{l}\text { NYHA class III or IV, } \\
\mathrm{n}(\%)\end{array}$ & $19(86.4 \%)$ & $57(80.3 \%)$ & 0.754 \\
\hline $\begin{array}{l}\text { Atrial fibrillation, } \mathrm{n} \\
(\%)\end{array}$ & $5(22.7 \%)$ & $15(21.1 \%)$ & 1.000 \\
\hline QRS > 150ms, n (\%) & $21(95.5 \%)$ & $64(90.1 \%)$ & 0.675 \\
\hline $\begin{array}{l}\text { QRS duration, mean } \\
( \pm \mathrm{SD})\end{array}$ & $158.2( \pm 14.7)$ & $160.1( \pm 22.5)$ & 0.636 \\
\hline non-LBBB, n (\%) & $1(4.5 \%)$ & $5(7.0 \%)$ & 1.000 \\
\hline $\begin{array}{l}\text { Myocardial infarction, } \\
\mathrm{n}(\%)\end{array}$ & $2(9.1 \%)$ & $10(14.1 \%)$ & 0.725 \\
\hline Stroke, n (\%) & $3(13.6 \%)$ & $4(5.6 \%)$ & 0.350 \\
\hline $\begin{array}{l}\text { Hemoglobin, g/dL } \\
( \pm \mathrm{SD})\end{array}$ & $11.8( \pm 3.1)$ & $12.8( \pm 1.8)$ & 0.147 \\
\hline $\begin{array}{l}\text { Chronic kidney disease, } \\
\mathrm{n}(\%)\end{array}$ & $8(36.4 \%)$ & $17(23.9 \%)$ & 0.278 \\
\hline Diabetes, n (\%) & $7(31.8 \%)$ & $23(32.4 \%)$ & 1.000 \\
\hline \multicolumn{4}{|l|}{ Medications } \\
\hline ACEI, ARB or ARNI & $20(90.9 \%)$ & $61(85.9 \%)$ & 0.725 \\
\hline Beta-blocker & $21(95.5 \%)$ & $65(91.5 \%)$ & 1.000 \\
\hline Spironolactone & $18(81.8 \%)$ & $63(88.7 \%)$ & 0.469 \\
\hline
\end{tabular}

LVEF, left ventricular ejection fraction; LBBB, left bundle branch block; NYHA, New York Heart Association; ARNI, angiotensin receptor neprilysin inhibitor;

ACEI, angiotensin-converting enzyme inhibitors; ARB, angiotensin receptor blockers; SD, standard deviation.

Table 2: Univariate and multivariate predictors of death from any cause

Univariate analysis Multivariate analysis

\begin{tabular}{lllll}
\hline & HR $(\mathbf{9 5 \%} \mathbf{C I})$ & $\mathbf{P}$ & HR $(\mathbf{9 5 \%} \mathbf{C I})$ & $\mathbf{P}$ \\
\hline Age, years & $1.0(0.9-1.0)$ & 0.808 & - & - \\
Stroke & $1.2(0.3-4.9)$ & 0.839 & - & -
\end{tabular}




\begin{tabular}{lllll}
\hline & HR $\mathbf{( 9 5 \%} \mathbf{C I})$ & $\mathbf{P}$ & HR $\mathbf{( 9 5 \%} \mathbf{C I})$ & $\mathbf{P}$ \\
\hline NYHA class III or IV & $1.2(0.4-2.8)$ & 0.354 & - & - \\
LVEF, baseline (\%) & $0.9(0.9-1.0)$ & 0.335 & - & - \\
QRS duration (ms) & $1.0(0.9-1.0)$ & 0.648 & - & - \\
Chronic kidney disease & $0.7(0.3-1.7)$ & 0.510 & - & - \\
Hemoglobin (g/dL) & $1.0(0.8-1.2)$ & 0.657 & - & - \\
Atrial fibrillation & $0.9(0.4-2.2)$ & 0.895 & - & - \\
CRT-D & $0.6(0.2-1.0)$ & 0.055 & $0.7(0.3-1.4)$ & 0.283 \\
Chagas cardiomyopathy & $3.9(1.8-8.4)$ & 0.001 & $2.1(0.9-5.3)$ & 0.106 \\
Ischemic cardiomyopathy & $2.1(0.6-7.0)$ & 0.224 & - & - \\
Upgrade & $4.7(2.2-9.9)$ & $<0.001$ & $2.9(1.2-7.1)$ & 0.019 \\
\hline
\end{tabular}

LVEF, Left ventricular ejection fraction; NYHA, New York Heart Association; CRT-D, Cardiac resynchronization therapy with defibrillator

Figure 1 - Kaplan-Meier estimates of survival by implantation type. CI indicates confidence interval; and HR, hazard ratio.

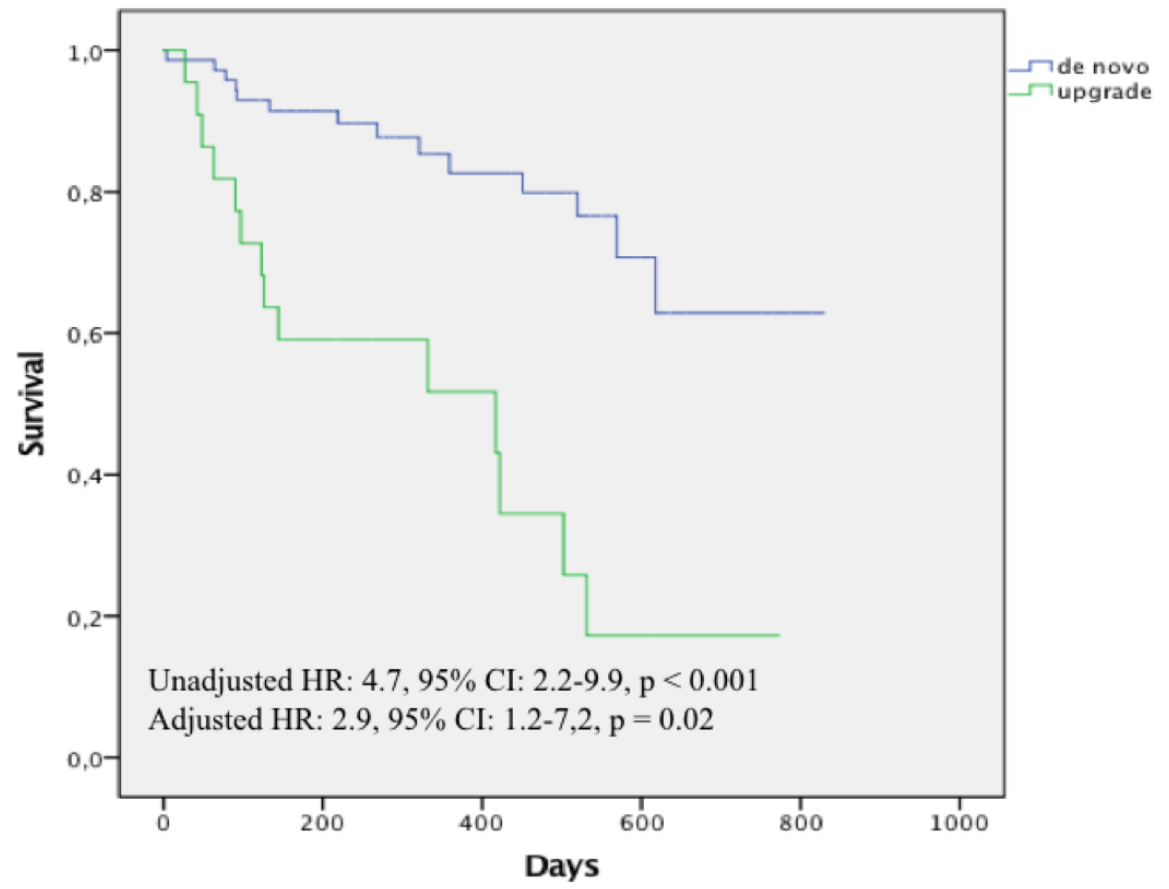

